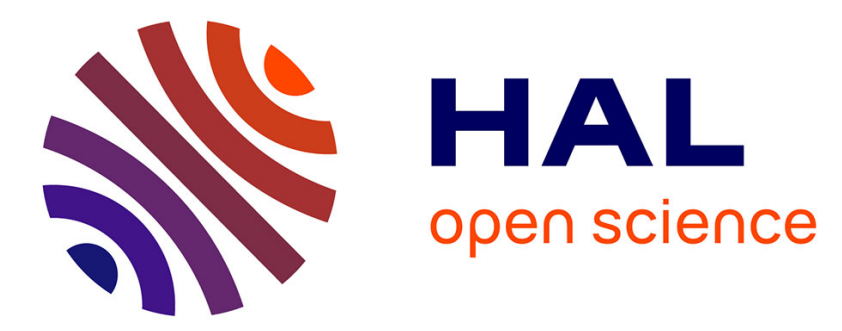

\title{
On Liveness and Deadlockability in Subclasses of Weighted Petri Nets
}

Thomas Hujsa, Raymond Devillers

\section{To cite this version:}

Thomas Hujsa, Raymond Devillers. On Liveness and Deadlockability in Subclasses of Weighted Petri Nets. 38th International Conference, PETRI NETS 2017, Zaragoza, Spain, June 25-30, 2017, Proceedings, pp.267-287, 2017. hal-02127837

\section{HAL Id: hal-02127837 https://hal.science/hal-02127837}

Submitted on 13 May 2019

HAL is a multi-disciplinary open access archive for the deposit and dissemination of scientific research documents, whether they are published or not. The documents may come from teaching and research institutions in France or abroad, or from public or private research centers.
L'archive ouverte pluridisciplinaire HAL, est destinée au dépôt et à la diffusion de documents scientifiques de niveau recherche, publiés ou non, émanant des établissements d'enseignement et de recherche français ou étrangers, des laboratoires publics ou privés. 


\title{
On Liveness and Deadlockability in Subclasses of Weighted Petri Nets
}

\author{
Thomas Hujsa $(\varangle)^{1}$ and Raymond Devillers ${ }^{2}$ \\ 1 Department of Computing Science, Carl von Ossietzky Universität Oldenburg, \\ D-26111 Oldenburg, Germany \\ thomas.hujsa@uni-oldenburg.de \\ 2 Département d'Informatique, Université Libre de Bruxelles, Belgium \\ rdevil@ulb.ac.be
}

\begin{abstract}
Structural approaches have greatly simplified the analysis of intractable properties in Petri nets, notably liveness. In this paper, we further develop these structural methods in particular weighted subclasses of Petri nets to analyze liveness and deadlockability, the latter property being a strong form of non-liveness.

For homogeneous join-free nets, from the analysis of specific substructures, we provide the first polynomial-time characterizations of structural liveness and structural deadlockability, expressing respectively the existence of a live marking and the deadlockability of every marking.

For the join-free class, assuming structural boundedness and leaving out the homogeneity constraint, we show that liveness is not monotonic, meaning not always preserved upon any increase of a live marking.

Finally, we use this new material to correct a flaw in the proof of a previous characterization of monotonic liveness and boundedness for homogeneous asymmetric-choice nets, published in 2004 and left unnoticed.
\end{abstract}

Keywords: Structural analysis - Weighted Petri net - Deadlockability - Liveness · Boundedness · Monotonicity · Fork-attribution · Join-free · Communication-free $\cdot$ Synchronization-free $\cdot$ Asymmetric-choice.

\section{Introduction}

Liveness is a behavioral property of Petri nets that is fundamental for many real world applications, notably embedded and flexible manufacturing systems. Such applications have to keep all their functions (transitions) active over time, a condition modeled by the liveness property. Deadlockability states the existence of a reachable deadlock (i.e. a dead marking, from which no transition is fireable) and is a particular case of non-liveness; its negation, deadlock-freeness, is often studied together with liveness.

Importance of weights. In this paper, we investigate weighted Petri nets, which are well suited to the modeling of real-life systems. In the domain of embedded systems, Synchronous Data Flow graphs [12] have been introduced 
to model the communications between a finite set of periodic processes. These graphs can be modeled by weighted T-nets, a Petri net subclass in which each place has at most one input and one output. In the domain of flexible manufacturing systems (FMS), the weights make possible the modeling of bulk consumption or production of resources [20]. In these cases, weights allow a compact representation of the volumes of data or resources exchanged.

Relationship with boundedness. Embedded applications have to use a limited amount of memory, a requirement formalized by the notion of boundedness in Petri nets. An objective is to extend the expressiveness of weighted T-nets so as to model more complex embedded applications while ensuring liveness and boundedness efficiently.

Analysis, subclasses and structure. Although decidable $[7,6]$, the problems of checking liveness or boundedness are EXPSPACE-hard $[7,4,13]$. A common approach to alleviate this difficulty is to consider specific subclasses and to relate the structure of the net to its behavior.

Homogeneity of weights is a restriction that simplifies the study of weighted classes: a net is homogeneous if each place has all its outputs weights equal [22, 11]. In this paper, we focus on join-free (JF) Petri nets, which forbid synchronizations, and homogeneous asymmetric-choice (HAC) nets, in which each pair $p, p^{\prime}$ of input places of any synchronization satisfies the following: all the outputs of $p$ are also outputs of $p^{\prime}$, or conversely. HAC nets generalize weighted T-nets and homogeneous JF (HJF) nets.

Many efforts have been devoted to the structural analysis of Petri nets, yielding efficient checking methods in particular subclasses. Structural liveness states the existence of a live marking, while structural boundedness ensures boundedness for every initial marking. Polynomial-time characterizations of both properties are known for ordinary (unit-weighted) free-choice (OFC) nets (in which all conflicting transitions have equal enabling conditions), based on decompositions into specific subnets (e.g. siphons and traps) and inequalities on the rank of the incidence matrix of the net (Rank theorems) $[2,5,18]$. From such structural conditions, polynomial-time methods checking the liveness of an initial marking have been deduced for bounded OFC nets [5].

Similar techniques, sometimes in a weaker form, have been developed for other classes with weights, including JF and HAC nets $[22,16,11,1,20,18,9]$.

Monotonic behavior. Another crucial criterion is the monotonicity of desired properties, meaning their preservation upon any increase of the initial marking. Embedded and manufacturing systems, among others, need their behavior to be maintained regardless of any addition of initial data items or resources. Liveness and boundedness are not monotonic in general, even when taken together, e.g. in HAC nets $[8,18]$. However, monotonic liveness (m-liveness) is fulfilled by OFC nets and some larger classes that contain HJF nets $[5,22,16,2,3,1]$.

Several complex Petri net subclasses are decomposable into specific JF subnets induced by subsets of places. In the HAC class and its subclass of homo- 
geneous (weighted) free-choice (HFC) nets, m-liveness has been expressed in terms of the m-liveness of such JF substructures [22,11]. Exploiting this fact in a bottom-up approach, polynomial-time sufficient conditions of m-liveness for bounded JF nets were shown to propagate to the decomposable, bounded HFC nets [10]. For the larger class of weighted free-choice nets, which contains HFC nets, there exist polynomial-time sufficient conditions of decomposability into structurally live and bounded JF nets [22].

Moreover, in any m-live system, every subsystem induced by any subset of places is necessarily m-live $[3,11,8]$. Hence, JF subnets form basic modules of major importance for the study of liveness in decomposable classes.

Contributions. For the structural liveness analysis of HJF nets, we highlight the importance of sub-consistency, which states the existence of a positive vector whose left-multiplication by the incidence matrix yields a non-null vector with no positive component. We use this algebraic notion to develop the first polynomial time characterizations of structural deadlockability (meaning deadlockability of every marking) and structural liveness for HJF nets, without the classical assumption of structural boundedness (or conservativeness) exploited in previous studies $[5,22,18]^{3}$.

To achieve it, we first restrict our attention to siphons, i.e. subsets $S$ of places satisfying the next property on their surrounding transitions: the input set of $S$ is included in the output set of $S$. More precisely, in any HJF net whose set of places is its unique siphon, we show the following: sub-consistency is equivalent to structural deadlockability, and non-sub-consistency is equivalent to structural liveness. Since sub-consistency can be checked with a linear program, these conditions can be evaluated in polynomial-time. Also, we extend this result to the rest of the HJF class, using a decomposition into minimal siphons.

Then, leaving out homogeneity, we show that live, structurally bounded JF nets are not always m-live, in constrast with the homogeneous case [22].

Finally, we use this new material to correct an erroneous proof of a previous characterization of m-liveness-boundedness for HAC nets, published in 2004 in [11] and left unnoticed.

Organisation of the paper. We formalize in Section 2 the notions used in this paper. In Section 3, we present the polynomial-time conditions for the structural deadlockability and liveness of HJF nets. We show in Section 4 that m-liveness does not apply to all bounded JF nets. In Section 5, we correct the proof of the mentioned previous result on the m-liveness-boundedness of HAC nets. Finally, Section 6 presents our conclusion and perspectives.

\section{Definitions, Notations and Properties}

In this section, we present the main notions used in the paper.

\footnotetext{
${ }^{3}$ Moreover, the well-known necessary conditions of liveness based on siphons containing traps or based on the existence of a repetitive vector $[5,18,3]$ do not help.
} 


\subsection{Weighted and Ordinary Nets}

A (weighted) net is a triple $N=(P, T, W)$ where the sets $P$ and $T$ are finite and disjoint, $P$ is the set of places, $T$ is the set of transitions, and $W:(P \times T) \cup$ $(T \times P) \rightarrow \mathbb{N}$ is a weight function. $P \cup T$ is the set of the nodes of the net. An arc leads from a place $p$ to a transition $t$ (respectively from a transition $t$ to a place $p$ ) if $W(p, t)>0$ (respectively $W(t, p)>0$ ). An ordinary net is a net whose weight function $W$ takes its values in $\{0,1\}$.

The incidence matrix of a net $(P, T, W)$ is a place-transition matrix $\mathcal{I}$ such that $\forall p \in P, \forall t \in T, \mathcal{I}[p, t]=W(t, p)-W(p, t)$, where the weight of a nonexisting arc is 0 . The pre-set of element $x$ of $P \cup T$, denoted by $x$, is the set $\{w \mid W(w, x)>0\}$. By extension, for any subset $E$ of $P$ or $T, \bullet^{\bullet}=\bigcup_{x \in E} \bullet$. The post-set of element $x$ of $P \cup T$, denoted by $x^{\bullet}$, is the set $\{y \mid W(x, y)>0\}$. Similarly, $E^{\bullet}=\bigcup_{x \in E} x^{\bullet}$.

A join-transition is a transition having at least two input places. Such a transition represents a synchronization on its input places. A choice-place is a place having at least two output transitions.

\subsection{Markings, Systems, Firing Sequences and Reachability}

A marking $M$ of a net $N=(P, T, W)$ is a mapping $M: P \rightarrow \mathbb{N}$. The pair $(N, M)$ defines a system whose initial marking is $M$. The system $(N, M)$ enables a transition $t \in T$ if $\forall p \in \bullet, M(p) \geq W(p, t)$. The marking $M^{\prime}$ obtained from $M$ by firing the enabled transition $t$, denoted by $M \stackrel{t}{\rightarrow} M^{\prime}$, is defined as follows: $\forall p \in P, M^{\prime}(p)=M(p)-W(p, t)+W(t, p)$.

A firing sequence $\sigma$ of length $n \geq 1$ on the set of transitions $T$, denoted by $\sigma=t_{i_{1}} t_{i_{2}} \ldots t_{i_{n}}$ with $t_{i_{1}}, t_{i_{2}}, \ldots, t_{i_{n}} \in T$, is a mapping $\{1, \ldots, n\} \rightarrow T$. The firing sequence $\sigma$ is feasible in $\left(N, M_{0}\right)$ if the successive markings obtained, $M_{0} \stackrel{t_{i_{1}}}{\longrightarrow} M_{1} \stackrel{t_{i_{2}}}{\longrightarrow} M_{2} \ldots \stackrel{t_{i_{n}}}{\longrightarrow} M_{n}$, are such that $M_{k-1}$ enables the transition $t_{i_{k}}$ for each $k \in\{1, \ldots, n\}$. We denote $M_{0} \stackrel{\sigma}{\rightarrow} M_{n}$.

The Parikh vector $\Psi(\sigma): T \rightarrow \mathbb{N}$ associated with a finite sequence of transitions $\sigma$ maps every transition $t$ of $T$ to the number of occurrences of $t$ in $\sigma$.

A marking $M^{\prime}$ is said to be reachable from the marking $M$ if there exists a firing sequence $\sigma$ feasible in $(N, M)$ such that $M \stackrel{\sigma}{\rightarrow} M^{\prime}$. The set of markings reachable from $M$ is denoted by $[M\rangle$.

\subsection{Petri Net Properties}

Main properties and monotonicity. Let $S=\left(N, M_{0}\right)$ be a system.

- A transition $t$ is dead in $S$ if no marking of $\left[M_{0}\right\rangle$ enables $t$. A deadlock, or dead marking, is a marking enabling no transition. $S$ is deadlock-free if no deadlock belongs to $\left[M_{0}\right\rangle$; otherwise it is deadlockable. The net $N$ is structurally deadlockable if, for every marking $M,(N, M)$ is deadlockable.

- A transition $t$ is live in $S$ if for every marking $M$ in $\left[M_{0}\right\rangle$, there is a marking $M^{\prime}$ in $[M\rangle$ enabling $t . S$ is live if every transition is live in $S . N$ is structurally live if a marking $M$ exists such that $(N, M)$ is live. 
- $S$ is bounded if an integer $k$ exists such that: $\forall M \in\left[M_{0}\right\rangle$, for each place $p$, $M(p) \leq k . N$ is structurally bounded if $(N, M)$ is bounded for each $M$.

- A behavioral property $\mathcal{P}$ is monotonic for $S$, or $S$ is monotonically $\mathcal{P}$, or $S$ is $m$ - $\mathcal{P}$, if $\left(N, M_{0}^{\prime}\right)$ satisfies $\mathcal{P}$ for every $M_{0}^{\prime} \geq M_{0}$. A marking $M$ is $m-\mathcal{P}$ if $(N, M)$ is $m-\mathcal{P}$, where $N$ is deduced from the context. We shall typically instantiate $\mathcal{P}$ with the liveness property.

Properties defined on nets extend to systems through their underlying net.

Vectors. The support of a vector $V$ with index set $I(V)$, noted $\mathcal{S}(V)$, is the set $\{i \in I(V) \mid V[i] \neq 0\}$ of indices of nonnull components. We denote by $\mathbb{0}^{n}$ (respectively $\mathbb{1}^{n}$ ) the column vector of size $n$ whose components are all equal to 0 (respectively 1 ). We may use the simpler notation $\mathbb{D}$ and $\mathbb{1}$ when $n$ is deduced from the context.

Conservativeness, consistency and variants. Let $N=(P, T, W)$ be a net with incidence matrix $\mathcal{I}$.

$-N$ is conservative if there exists a vector $X \geq \mathbb{1}$ such that $X^{T} \cdot \mathcal{I}=\mathbb{O}$.

$-N$ is consistent (respectively sur-consistent, sub-consistent) if there exists a vector $Y \geq \mathbb{1}$ such that $\mathcal{I} \cdot Y=\mathbb{O}$ (respectively $\mathcal{I} \cdot Y \gtrless \mathbb{O}, \mathcal{I} \cdot Y \lessgtr \mathbb{D}) . N$ is weakly sur-consistent (respectively weakly sub-consistent) if there exists a vector $Y \geq \mathbb{1}$ such that $\mathcal{I} \cdot Y \geq \mathbb{0}$ (respectively $\mathcal{I} \cdot Y \leq \mathbb{0}$ ), i.e. if it is consistent or sur-consistent (respectively consistent or sub-consistent). Weak sur-consistency is also known as structural repetitiveness.

- $N$ is partially consistent (respectively partially sur-consistent, partially subconsistent) if there exists a vector $Y \geqslant \mathbb{D}$ such that $\mathcal{I} \cdot Y=\mathbb{O}$ (respectively $\mathcal{I} \cdot Y \gtrless \mathbb{0}, \mathcal{I} \cdot Y \lessgtr 0)$.

\subsection{Petri Nets Subclasses}

A weighted net $N=(P, T, W)$ is:

- a $P$-net (or $S$-net) if $\forall t \in T:\left|{ }^{\bullet} t\right| \leq 1$ and $\left|t^{\bullet}\right| \leq 1$.

- a T-net (or generalized event graph) if $\forall p \in P:|\bullet p| \leq 1$ and $\left|p^{\bullet}\right| \leq 1$.

- join-free (JF) (or generalized communication-free, synchronization-free) if $\forall t \in T:|\bullet t| \leq 1$.

- choice-free (CF) (or output-nonbranching) if $\forall p \in P:\left|p^{\bullet}\right| \leq 1$.

- fork-attribution (FA) if it is both JF and CF.

- free-choice (FC) (or Topologically Extended Free Choice) if $\forall p_{1}, p_{2} \in P$, $p_{1}^{\bullet} \cap p_{2}^{\bullet} \neq \varnothing \Rightarrow p_{1}^{\bullet}=p_{2}^{\bullet}$. This class generalizes, with arbitrary weights, the ordinary free-choice nets (OFC) of the literature [5].

- asymmetric-choice (AC) if $\forall p_{1}, p_{2} \in P, p_{1} \bullet \cap p_{2} \bullet^{\bullet} \neq \emptyset \Rightarrow p_{1} \bullet \subseteq p_{2}{ }^{\bullet}$ or $p_{2}{ }^{\bullet} \subseteq p_{1}{ }^{\bullet}$. The class of AC nets contains the FA, CF, JF, and FC nets.

- homogeneous if, $\forall p \in P, \forall t, t^{\prime} \in p^{\bullet}, W(p, t)=W\left(p, t^{\prime}\right)$. The homogeneous subclass of a class is obtained with an additional prefix letter $\mathrm{H}$, e.g. HFC denoting the homogeneous FC nets.

Some illustrations for these classes are presented in Figure 1. 

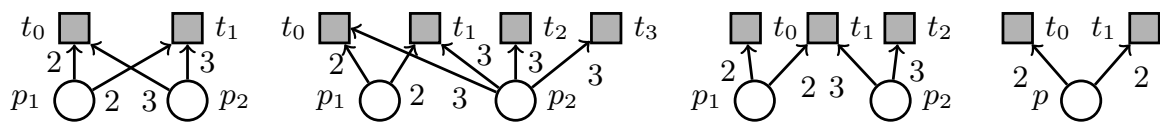

Fig. 1. The net on the left is HFC, the second one is HAC. The third net is homogeneous, non-AC since ${ }^{\bullet} t_{1}=\left\{p_{1}, p_{2}\right\}$, while $p_{1} \bullet \nsubseteq p_{2}{ }^{\bullet}$ and $p_{2} \bullet \nsubseteq p_{1} \bullet$. Since they have synchronizations, they are not JF. However, the fourth net is HJF.

\subsection{Graph Structures}

Subnets and subsystems. Let $N=(P, T, W)$ and $N^{\prime}=\left(P^{\prime}, T^{\prime}, W^{\prime}\right)$ be two nets. $N^{\prime}$ is a subnet of $N$ if $P^{\prime}$ is a subset of $P, T^{\prime}$ is a subset of $T$, and $W^{\prime}$ is the restriction of $W$ to $\left(P^{\prime} \times T^{\prime}\right) \cup\left(T^{\prime} \times P^{\prime}\right) . S^{\prime}=\left(N^{\prime}, M_{0}^{\prime}\right)$ is a subsystem of $S=\left(N, M_{0}\right)$ if $N^{\prime}$ is a subnet of $N$ and its initial marking $M_{0}^{\prime}$ is the restriction of $M_{0}$ to $P^{\prime}$, i.e. $M_{0}^{\prime}=\left.M_{0}\right|_{P^{\prime}}$.

$N^{\prime}$ is a $P$-subnet of $N$ if $N^{\prime}$ is a subnet of $N$ and $T^{\prime}=\bullet P^{\prime} \cup P^{\prime \bullet}$, the preand post-sets being taken in $N . S^{\prime}=\left(N^{\prime}, M_{0}^{\prime}\right)$ is a $P$-subsystem of $S=\left(N, M_{0}\right)$ if $N^{\prime}$ is a P-subnet of $N$ and $S^{\prime}$ is a subsystem of $S$.

Similarly, $N^{\prime}$ is a T-subnet of $N$ if $N^{\prime}$ is a subnet of $N$ and $P^{\prime}=\bullet T^{\prime} \cup T^{\bullet \bullet}$, the pre- and post-sets being taken in $N . S^{\prime}=\left(N^{\prime}, M_{0}^{\prime}\right)$ is a T-subsystem of $S=\left(N, M_{0}\right)$ if $N^{\prime}$ is a T-subnet of $N$ and $S^{\prime}$ is a subsystem of $S$.

Notice that a T-subnet (respectively P-subnet) is not necessarily a T-net (respectively P-net).

Siphons and traps. Consider a net $N=(P, T, W)$. A non-empty subset $D \subseteq P$ of places is a siphon (sometimes also called a deadlock) if $D \subseteq D^{\bullet}$. A non-empty subset $Q \subseteq P$ of places is a trap if $Q^{\bullet} \subseteq{ }^{\bullet} Q$.

Reduced graphs. The reduced graph $R$ of a net $N$ is the directed graph $G=(V, A)$ obtained from $N$ by contracting every maximal strongly connected component $c$ of $N$ into one single node $g_{c} \in V$. The set $A$ of arcs represents the connections that remain after the contraction: for any two distinct nodes $g_{u}, g_{u^{\prime}}$ of $R$ that represent respectively the distinct components $u, u^{\prime}$ of $N$, we have an $\operatorname{arc}\left(g_{u}, g_{u^{\prime}}\right)$ from $g_{u}$ to $g_{u^{\prime}}$ if $W\left(q, q^{\prime}\right)>0$ in $N$ for some $q \in u$ and $q^{\prime} \in u^{\prime}$. By definition, each reduced graph is acyclic. This is illustrated on Figure 2.
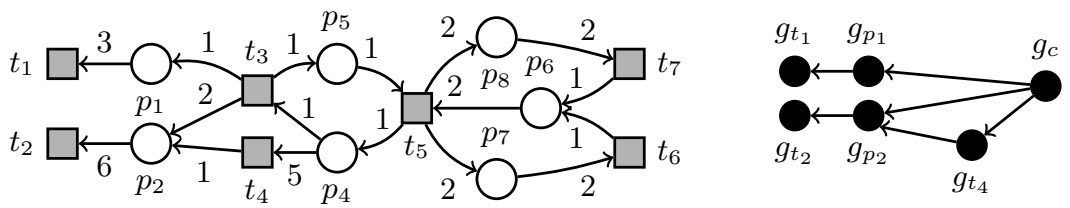

Fig. 2. On the right, the reduced graph represents the net on the left. The node $g_{c}$ represents the subnet $c$ defined by places $p_{4}, p_{5}, p_{6}, p_{7}, p_{8}$ and transitions $t_{3}, t_{5}, t_{6}, t_{7}$. 
In what follows, without loss of generality, we consider connected nets that contain at least a place and a transition, unless otherwise specified.

\section{Deadlockability and Liveness of Homogeneous JF Nets}

Liveness, deadlockability and related properties have been studied previously in the (weighted) join-free class, notably under the conservativeness (i.e. structural boundedness) assumption $[20,22,18,9]$ or in more restricted subclasses $[14,15]$.

In this section, we show that the notion of sub-consistency is a fundamental algebraic property characterizing structural deadlockability in the HJF nets covered by a (unique) minimal siphon ${ }^{4}$. As a corollary, we obtain for the same nets that structural liveness is equivalent to non-sub-consistency. Since subconsistency can be checked with linear programming, we deduce a polynomialtime method checking structural deadlockability or structural liveness. We then generalize these conditions to all HJF nets, by means of coverings with minimal siphons. These results are dedicated to the HJF structure and do not apply to inhomogeneous JF nets.

The characterization of structural deadlockability for the HJF nets covered by a unique (minimal) siphon arises from a series of intermediate results.

First, we study a general relationship between deadlockability, non-liveness and sub-consistency in the JF class.

Second, using previous results on the CF class, we show that strongly connected JF systems are covered by strongly connected FA T-subsystems that may either decrease, preserve or generate tokens in the JF system.

Third, we provide a variant of this classification, proving that sub-consistency is equivalent to structural deadlockability in strongly connected FA nets.

Fourth, we show that each strongly connected, sub-consistent JF net contains a strongly connected, sub-consistent (structurally deadlockable) FA T-subnet.

Finally, we prove by induction on the structure of the sub-consistent HJF nets covered by a unique siphon that every marking can reach a deadlock by directing tokens towards a sub-consistent, hence deadlockable, FA T-subsystem.

To simplify the development of these statements, we reveal and exploit the graph structures corresponding to minimal siphons in join-free nets (Lemma 1), namely quasi strongly connected nets, defined below.

Quasi-strong connectedness and siphons. A net is called quasi strongly connected if it is connected and becomes strongly connected once we omit the transitions with no output. We will use the next correspondence with siphons.

Lemma 1. Let $N=(P, T, W)$ be a connected JF net. Then, $P$ is the unique siphon of $N$ if and only if $N$ is quasi strongly connected.

\footnotetext{
${ }^{4}$ In this paper, we study siphons that may contain traps, remarkably in JF nets. Hence, we cannot use the results of [3]. Also, our nets will often be structurally repetitive (weakly sur-consistent), which is another well-known necessary condition of structural liveness (Prop. 10 in [18]) that is not sufficient in the HJF class.
} 
Proof. Assume that $P$ is the unique siphon of $N$. If $N$ is not quasi strongly connected, consider a maximal quasi strongly connected subnet $N^{\prime}$ without input node and containing a place (consider a node $g$ without input in the reduced graph of $N$; since $P$ is a non-empty siphon, $g$ is not a single transition and contains a place, while the subnet induced by the union of $g$ with its output transitions defines $N^{\prime}$ ). Since $N$ is not quasi strongly connected, the places of $N^{\prime}$ define a smaller siphon of $N$, a contradiction.

Conversely, if $N$ is quasi strongly connected, then $P$ is a siphon. Suppose that a smaller siphon $P^{\prime}$ exists. For any place $p \in P \backslash P^{\prime}$, and any directed path from $p$ to some place of $P^{\prime}$, every place of this path belongs to $P^{\prime}$ (by definition of siphons and by join-freeness). Since all places of a quasi strongly connected net belong to the same unique maximal strongly connected component of the net, $P^{\prime}=P$, a contradiction. Hence, $P$ is the unique siphon of $N$.

\subsection{Relating Deadlockability to Non-liveness and Sub-consistency}

We provide next a necessary condition for structural deadlockability in JF nets.

Lemma 2. Let $N$ be a JF net in which every place $p$ has at least one output transition. If $N$ is structurally deadlockable, it is sub-consistent.

Proof. One can choose a sufficiently large marking $M_{0}$, with $M_{0}(p) \geq W(p, t)$ $\forall p \in P, \forall t \in p^{\bullet}$, that enables a sequence $\sigma$ containing all transitions, leading to a marking $M$. A sequence $\sigma^{\prime}$ is feasible at $M$ that leads to a deadlock $M^{\prime}$. From join-freeness, for each place $p$ and each output transition $t$ of $p$, we have $M^{\prime}(p)<$ $W(p, t)$. Let us define $\tau=\sigma \sigma^{\prime}$. The Parikh vector $\Psi(\tau)$ satisfies $\Psi(\tau) \geq \mathbb{1}$. Moreover, for every place $p, M^{\prime}(p)<M_{0}(p)$, from which we deduce $\mathcal{I} \cdot \Psi(\tau) \leq-\mathbb{1}$, where $\mathcal{I}$ is the incidence matrix of $N$. Thus, $N$ is sub-consistent.

The converse does not hold: the ordinary HJF net formed of two transitions $t, t^{\prime}$, a place $p$ and two unit-weighted arcs such that $t$ is the input of $p$ and $t^{\prime}$ is its output, is sub-consistent (look at $\Psi\left(t t^{\prime} t^{\prime}\right)$ ) and live for every initial marking.

The next equivalence between liveness and deadlock-freeness is inspired from $[21,22]$, restricted to HJF nets but extended to possibly unbounded nets.

Lemma 3. Let $S=\left(N, M_{0}\right)$ be a quasi strongly connected HJF system. $S$ has a non-live transition if and only if it is deadlockable.

Proof. As usual, we consider nets with $T \neq \emptyset$. It is clear that the reachability of a deadlock implies non-liveness. Now, if a transition $t$ is dead in some reachable marking $M$, all transitions in $\left(\bullet^{\bullet} t\right)^{\bullet}$ are also dead in $M$ since the net is HJF, and since $|\bullet t|=1$ we deduce that each transition in $\bullet^{\bullet} t$ can be fired only a finite number of times from $M$, leading to a marking at which these transitions are dead. One can iterate this process on each directed path that reaches some dead transition, leading to a deadlock by quasi strong connectedness.

The next lemma relates structural deadlockability to non-structural liveness in the systems for which liveness is equivalent to deadlock-freeness. 
Lemma 4. Let $N$ be a net such that, for every marking $M$ of $N$, deadlockfreeness of $(N, M)$ implies liveness of $(N, M)$. Then, $N$ is structurally deadlockable if and only if it is not structurally live.

Proof. Structural deadlockability obviously implies non-structural liveness for all Petri nets with $T \neq \emptyset$. For the converse, if $N$ is not structurally live, every marking $M$ is non-live for this net. Then, using the assumption that non-liveness of $(N, M)$ implies deadlockability of $(N, M)$, we deduce that a deadlock is reachable from every marking $M$, implying structural deadlockability of $N$.

This result also applies to quasi strongly connected HJF nets by Lemma 3.

\subsection{Previous Results Relating JF and CF Nets to their FA Subclass}

Basing on previous works on the FA and CF classes, we exhibit fundamental structural and behavioral properties of JF nets, expressed in terms of FA nets: strongly connected JF nets are covered by strongly connected FA T-subnets, the latter benefitting from a structural classification for liveness and boundedness. Roughly speaking, actions performed in such FA T-subnets may either decrease, preserve or generate tokens in the associated area of the JF system.

Reverse-duality and covering. Structural results may be obtained directly from known properties of the reverse-dual net, which is defined by reversing arcs and swapping places with transitions. This method has been used for CF nets, whose reverse-dual class is the JF class [20]. However, in general, the relationship between the structure of a net and its behavioral properties cannot be deduced from known properties of the reverse-dual net. Using reverse-duality, we obtain the next variation of Lemma 10 in [20], getting a first glimpse of the important role played by particular FA T-subnets in the behavior of JF nets since any sequence feasible in a T-subsystem is feasible in the system.

Lemma 5 (Reverse-dual of Lemma 10 in [20]). Let $N$ be a strongly connected JF net. For each transition $t$ of $N$, there is a strongly connected FA T-subnet of $N$ containing $t$.

In the following, we link the structure of FA nets to their behavior.

Structural liveness of CF nets. We deduce from Corollary 4 in [20] the next characterization of structural liveness for CF nets, which generalize FA nets.

Lemma 6. A CF net is structurally live if and only if it is weakly sur-consistent.

A previous classification of strongly connected FA nets. Strongly connected FA nets have been previously studied, notably in $[19,20]$ where they are presented as a natural generalization of weighted circuits. In the same studies (page 6 in [20] and Section 4.1 in [19]), it is explained that the class of strongly connected FA nets can be partitioned into three subclasses: "neutral", when the 

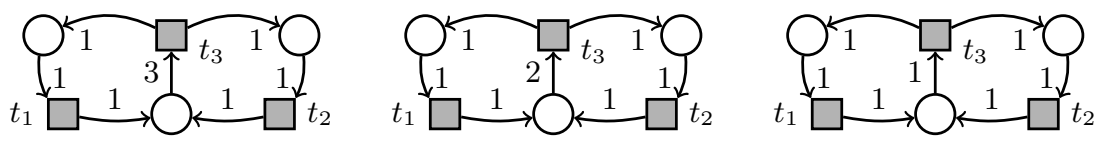

Fig. 3. From left to right: an absorbing, a neutral and a generating FA net.

FA net is consistent and conservative; "absorbing", when the FA net is not weakly sur-consistent; or "generating", when the FA net is not structurally bounded. Figure 3 depicts an element of each class.

This partition does not apply to strongly connected JF nets, even if one tries to replace non-weak sur-consistency by sub-consistency and even for their subclass of homogeneous P-nets, as shown in Figure 4.

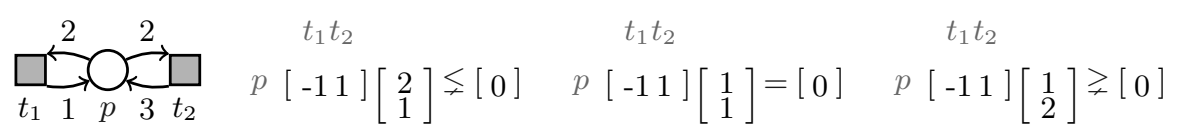

Fig. 4. The inequalities on the right show the homogeneous P-net on the left to be jointly sub-consistent, consistent and sur-consistent. Hence, these three properties cannot be used alone to tri-partition the class of strongly connected homogeneous P-nets.

Hence, this classification is tightly related to the FA structure. We will show in Lemma 12 that non-weak sur-consistency is equivalent to sub-consistency in strongly connected FA nets.

\subsection{Small Nets in Petri Nets and Sub-consistency in JF Nets}

We present below the notion of small nets and introduce associated results about decompositions into T-subnets. From this development, we express a variant of the previous classification of FA nets in terms of sub-consistency, and deduce the existence of a strongly connected sub-consistent FA T-subnet in every strongly connected sub-consistent JF net.

In the sequel, the general type $\mathcal{P}$ used in the next definition shall be specialized to consistency, sur-consistency or sub-consistency.

Definition 1 ([17]). A net of type $\mathcal{P}$ is said to be small $\mathcal{P}$ if it does not contain any non-empty proper $T$-subnet of the same type $\mathcal{P}$.

In strongly connected, partially sub-consistent JF nets, the strong connectedness of small sub-consistent T-subnets is revealed next.

Lemma 7. Let $N$ be a strongly connected, partially sub-consistent JF net. Then, every small sub-consistent T-subnet of $N$ is strongly connected. Moreover, there exists such a (non-empty) strongly connected T-subnet in $N$. 
Proof. Denote by $\mathcal{I}$ the incidence matrix of $N$. By partial sub-consistency, there exists a vector $\pi \gtrless \mathbb{O}$ such that $\mathcal{I} \cdot \pi \lessgtr \mathbb{O}$. Let us denote by $J$ any small subconsistent T-subnet of $N$. Such a subnet exists, since the T-subnet $N_{\pi}$ of $N$ induced by the support of $\pi$ is sub-consistent. We show that $J$ is necessarily strongly connected. Suppose that $J$ is not strongly connected. If $J$ is not connected, it contains a proper sub-consistent T-subnet, contradicting the fact that $J$ is small sub-consistent. Hence, $J$ is connected.

Consider the reduced graph $R$ of $J$. It is acyclic (so that it defines a partial order), connected (since so is $J$ ) and finite (so that there are maximal nodes). Let us consider any such maximal node $g$, meaning that $g$ has some input in $R$ (otherwise $g$ would be $R$ and $J$ would be strongly connected) but no output in $R$. We show that the subgraph corresponding to $g$ is a strongly connected T-subnet of $N$ that contains at least a place and a transition. By definition, $g$ contains a node and is strongly connected.

If $g$ is a single place, then it has an input transition in $J$ and no output in $J$, so that a sub-consistency vector $\mu_{J}$ for $J$ cannot yield a null or negative value (when left-multiplied by the incidence matrix $\mathcal{I}_{J}$ of $J$ ) for this place, a contradiction. If $g$ is a single transition, it has no output in $J$, hence no output in $N$ since $J$ is a T-subnet of $N$, contradicting the strong connectedness of $N$.

Thus, $g$ contains at least a place and a transition. If $g$ is not a T-subnet of $J$, this means that it contains a transition $t$ lacking an input or an output. The transition $t$ must have its (unique, since $N$ is JF) input place in $g$, since $g$ is strongly connected. It has all its outputs in $g$ too, since $g$ has no output in $R$ (hence in $J \backslash g$ ) by the choice of $g$. We obtain a contradiction, implying that $g$ is a T-subnet of $J$ and $N$.

Now, we show that $g$ is sub-consistent. Denote by $\mathcal{I}_{J}$ the sub-matrix of $\mathcal{I}$ restricted to $J$. Since $J$ is sub-consistent, we have $\mathcal{I}_{J} \cdot \mu_{J} \lesseqgtr \mathbb{O}$ for some integer vector $\mu_{J} \geq \mathbb{1}$. Since $g$ has some input node in $R$, it has some input node $n$ in $J$, and since $g$ is strongly connected while $J$ is JF, $n$ must be a transition. Because $g$ is a maximal strongly connected component of $J$, any input transition of (a place of) $g$ cannot have its input place in $g$. Denote by $g^{\prime}$ the union of the net represented by $g$ and all its input transitions in $J$ with the arcs going from these inputs to $g$. (Notice that $g^{\prime}$ is a P-subnet of $J$.) Denote by $\mu_{g}$ and $\mu_{g}^{\prime}$ the restriction of $\mu_{J}$ to (the transitions of) $g$ and $g^{\prime}$ (so that $\mu_{g}, \mu_{g}^{\prime} \geq \mathbb{1}$ ) and by $\mathcal{I}_{g}$, $\mathcal{I}_{g}^{\prime}$ the incidence matrices of $g, g^{\prime}$ respectively. Since $g$ has no output in $R$ (nor in $J$ ), hence in particular no output transition in $J$, we have $\mathcal{I}_{g}^{\prime} \cdot \mu_{g}^{\prime} \leq \mathbb{O}$ (we may have equality if the negative values correspond to places not in $g$ ). Let $p$ be a place of $g$ that is an output of $n$. Then, $\left(\mathcal{I}_{g} \cdot \mu_{g}\right)(p)<0$, and $g$ is sub-consistent.

$J$ thus contains a proper T-subnet of the same type, i.e. a sub-consistent one, whereas $J$ is small sub-consistent, a contradiction. We deduce that $J$ is strongly connected, hence the claimed property.

This result is tightly related to the JF structure and does not apply to Petri nets with synchronizations, as illustrated in Figure 5.

The next result investigates small sub-consistent FA nets. 

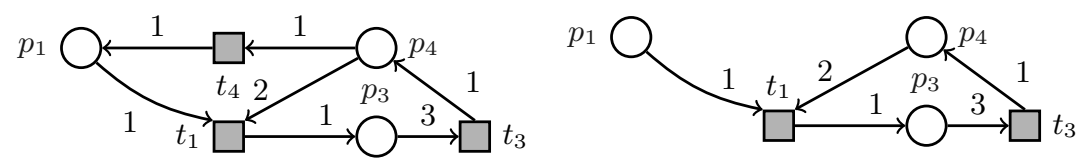

Fig. 5. The net on the left is strongly connected, sub-consistent and is not JF. On the right, its unique non-empty, small, sub-consistent T-subnet, is neither strongly connected nor JF.

Lemma 8. Let $N$ be a strongly connected $F A$ net. Then, $N$ does not contain any non-empty proper strongly connected T-subnet. Moreover, if $N$ has the property of sub-consistency it is small for this property.

Proof. Let us assume that $N$ contains a non-empty proper strongly connected T-subnet $F$. Hence, there exists a node in $N \backslash F$. Since $N$ is strongly connected, there exists a node $n$ in $N \backslash F$ that is an output of a node $n^{\prime}$ in $F$. The node $n^{\prime}$ cannot be a place (since $F$ is strongly connected and $n^{\prime \bullet}=\{n\}$, we should have that $n^{\prime}$ is the only node of $F$, but then $F$ is not a T-subnet). Thus, $n^{\prime}$ is a transition and $n$ is an output place of $n^{\prime}$ that is not in $F$, contradicting the fact that $F$ is a T-subnet.

If $N$ is sub-consistent, Lemma 7 applies, implying that every small subconsistent T-subnet of $N$ is strongly connected, and there is a non-empty one in $N$; from the first part of the claim, we deduce that $N$ is small sub-consistent.

It can be seen that the second part of the above result becomes false when sur-consistency is considered instead of sub-consistency.

Structural deadlockability and sub-consistency in FA nets. To characterize structural deadlockability in FA nets, we need the next technical result.

Lemma 9 (Propositions 16a, 18a in [17]). If a net is small sur-consistent, then it does not contain any consistent or sub-consistent T-subnet (either proper or not). If a net is small sub-consistent, then it does not contain any consistent or sur-consistent T-subnet (either proper or not).

We are now able to deduce the following necessary and sufficient condition.

Lemma 10. Let $N$ be any strongly connected $F A$ net. $N$ is structurally deadlockable if and only if it is sub-consistent.

Proof. If $N$ is sub-consistent, it is small sub-consistent (by Lemma 8). By Lemma 9 it is neither consistent nor sur-consistent, hence it is not weakly sur-consistent. By Lemma 6, it is not structurally live. By Lemma 4, it is structurally deadlockable. For the converse, suppose that $N$ is structurally deadlockable. Then, Lemma 2 applies. 
A variant of the classification of strongly connected FA nets. By relating sub-consistency to structural deadlockability and non-weak sur-consistency, we obtain a variant of the classification of $[19,20]$. To achieve it, we need the next lemma.

Lemma 11. Consider any net $N=(P, T, W)$ with incidence matrix $\mathcal{I}$ having a consistency vector $\pi_{1}$ and a vector $\pi \geq \mathbb{0}$ such that $\mathcal{I} \cdot \pi \lessgtr \mathbb{0}$ or $\mathcal{I} \cdot \pi \geq \mathbb{0}$. Then, in both cases, $N$ is sub-consistent and sur-consistent.

Proof. Define $\pi^{\prime}=\pi_{1}+\pi$, so that $\pi^{\prime} \geq \mathbb{1}$, and $\pi^{\prime \prime}=k \cdot \pi_{1}-\pi$, where $k$ is any (sufficiently large) positive integer such that $\pi^{\prime \prime} \geq \mathbb{1}$. If $\mathcal{I} \cdot \pi \leq \mathbb{0}$, then $\pi^{\prime}$ is a sub-consistency vector for $N$ and $\pi^{\prime \prime}$ is a sur-consistency vector. Similarly, if $\mathcal{I} \cdot \pi \gtrless \mathbb{O}, \pi^{\prime}$ is a sur-consistency vector and $\pi^{\prime \prime}$ is a sub-consistency vector.

Lemma 12. Let $N$ be a strongly connected FA net. $N$ satisfies exactly one of the following properties: consistency, sub-consistency or sur-consistency.

Proof. For this class, structural liveness is equivalent to weak sur-consistency (consistency or sur-consistency) (Lemma 6), while non-structural liveness is equivalent to structural deadlockability (Lemma 4) and sub-consistency (Lemma 10). We deduce that this class can be partitioned into two subclasses: the subconsistent ones and the weakly sur-consistent ones. If $N$ is both consistent and sur-consistent, it is also sub-consistent (by Lemma 11), a contradiction with the previous observation. We deduce the claim.

Existence of strongly connected sub-consistent FA T-subnets. Using the new classification, the next refinement of Lemma 7 reveals the FA structure.

Lemma 13. Let $N$ be a strongly connected, partially sub-consistent JF net. Then, every small sub-consistent T-subnet of $N$ is a strongly connected FA net. Moreover, $N$ contains such a (non-empty) strongly connected FA T-subnet.

Proof. Applying Lemma 7, every small sub-consistent T-subnet of $N$ is strongly connected, and $N$ contains such a non-empty T-subnet $J$. Suppose that $J$ is not FA. By Lemma 5 , we know that $J$ is covered by strongly connected FA Tsubnets. Since $J$ is small sub-consistent, none of them is sub-consistent. Then, using the new classification (Lemma 12), each of them is either consistent or sur-consistent. By Lemma 9, $J$ does not contain any consistent T-subnet nor any sur-consistent T-subnet. We obtain a contradiction, and $J$ is FA.

\subsection{Polynomial-time Intermediary Characterizations}

In the following, we provide two characterizations of structural deadlockability and liveness for the HJF nets covered by a unique siphon, or equivalently quasi strongly connected HJF nets by Lemma 1 .

The next theorem investigates structural deadlockability. The main step of the proof is illustrated in Figure 6. 
Theorem 1 (Structural deadlockability). Consider any quasi strongly connected HJF net. It is sub-consistent if and only if it is structurally deadlockable.

Proof. Structural deadlockability and Lemma 2 imply sub-consistency. We prove the other direction by induction on the number $n$ of places with several outputs.

Base case: $n=0$. If $N$ does not contain any choice, it is either a subconsistent strongly connected FA net, or a single output-free transition together with its unique input place. In the first case, Lemma 10 applies, from which we deduce structural deadlockability. We see easily that the second case also implies structural deadlockability.

Inductive case: $n>0 . N$ contains choices: it is not an FA net. We suppose that the claim is true for every $n^{\prime}<n$. If $N$ is strongly connected, Lemma 13 applies, and $N$ contains a sub-consistent, strongly connected FA T-subnet $F$. Otherwise, $N$ contains an output-free transition, and we denote by $F$ the T-subnet formed of this transition with its unique input place.

Let $N^{\prime}$ be the subnet of $N$ obtained by deleting all the places of $F$ and their outgoing transitions. If $N^{\prime}$ is empty, then all the places of $N$ belong to $F$, in which case firing only in $F$ leads to a deadlock (by Lemma 10 if $F$ is strongly connected, trivially in the case of two nodes), since the net is homogeneous. Otherwise, in the rest of the proof, we suppose that $N^{\prime}$ is not empty.

Let $N_{1}, \ldots, N_{k}$ be all the maximal connected (not necessarily strongly connected) components of $N^{\prime}$. In the following, we prove these components to be structurally deadlockable. Consider any such net $N_{i}$. It cannot contain a transition without input, since such transitions do not occur in $N$ and all outputs of the deleted places were deleted. Thus, since it is not empty, it contains a place.

Since all places of $N$ belong to a same unique maximal strongly connected component of $N$, there is in $N$ a directed path from a place of $F$ to any place in $N_{i}$, and reciprocally. Consequently, there is a deleted node $u$ (not in $N_{i}$ ) input of some node in $N_{i}$. Since each transition of $N_{i}$ has an input place in $N_{i}$, and $N$ is JF, $u$ is a transition. By definition of $N^{\prime}$, the input place of $u$, and each input place of every other transition of the same kind, are deleted places and cannot belong to $N_{i}$. Moreover, all the transitions of $N$ that are outputs of places of $N_{i}$ have not been deleted and belong to $N_{i}$, since otherwise their input would have been deleted. Let $\mathcal{I}$ be the incidence matrix of $N$ and $\pi$ be a sub-consistency vector for $N$. In the sequel, each union of a transition $t$ with a subnet $g$ of a net $h$ is a net containing $g, t$ and all $\operatorname{arcs}$ between $t$ and $g$ in $h$. We have two cases.

First case. Suppose that $N_{i}$ is quasi strongly connected. A transition $t$ exists in $N$ that has been deleted and is an input of $N_{i}$, the input of $t$ not being in $N_{i}$. Also recall that $N_{i}$ has no output transition in $N \backslash N_{i}$. Denote by $N_{i}^{\prime}$ the union of $N_{i}$ with its deleted input transitions (and the arcs from these transitions to $N_{i}$ ). If $\mathcal{I}_{i}^{\prime}$ (respectively $\mathcal{I}_{i}$ ) is the incidence matrix of $N_{i}^{\prime}$ (respectively $N_{i}$ ), the projection $\pi_{i}^{\prime}$ of $\pi$ to $N_{i}^{\prime}$ satisfies $\mathcal{I}_{i}^{\prime} \cdot \pi_{i}^{\prime} \leq 0$. Denoting by $\pi_{i}$ the projection of $\pi$ to $N_{i}$, we deduce that $\mathcal{I}_{i} \cdot \pi_{i} \lesseqgtr 0: N_{i}$ is sub-consistent. Since it is quasi strongly connected and contains strictly fewer choices than $N$, the induction hypothesis applies, and $N_{i}$ is structurally deadlockable. 
Second case. Otherwise, suppose that $N_{i}$ has a different structure; it is not strongly connected. Recall that $N_{i}$ does not contain any transition having no input. Denote by $R$ its reduced graph. Consider any node $g$ of $R$ that contains at least one place and one transition. If no such $g$ exists in $R$, which is acyclic, it is clear that $N_{i}$ is structurally deadlockable. We consider the next cases for $g$.

Case a. If $g$ has no input in $R$, it has some output in $R$, because $N_{i}$ is not strongly connected. $g$ has necessarily a deleted input transition whose input has been deleted. Similarly to the "First case", the union of $g$ with its deleted input transitions and its possible output transitions is a sub-consistent or consistent net. Thus, the union of $g$ with its possible outgoing transitions is a sub-consistent subnet of $N_{i}$ that is quasi strongly connected.

Case b. If $g$ has some input transition and some output in $R$, similarly to the previous cases, the union of $g$ with its possible output transitions satisfies sub-consistency and is quasi strongly connected.

Case c. If $g$ has some input transition in $R$ and no output in $R$, it may have only output places in $N$. Using similar arguments as before, $g$ satisfies sub-consistency and is quasi strongly connected.

In all cases, the induction hypothesis can be applied to the union of $g$ with its possible output transitions, which all belong to $N_{i}$. We deduce that every such subnet of $N_{i}$ is structurally deadlockable. By following the partial order defined by $R$ on its directed paths, starting from the smallest nodes of $R$ for this order (i.e. with no input in $R$ ), selecting arbitrary (homogeneous) choices if needed, every node of $R$ can be successively deadlocked, finally deadlocking $N_{i}$.

Thus, $N_{i}$ is always structurally deadlockable. Its possible inputs in $N$ are necessarily deleted transitions, while its possible outputs are necessarily deleted places. Since all deleted places belong to a structurally deadlockable T-subnet $F$ of $N$, all the tokens produced in such places when deadlocking all $N_{j}, 1 \leq j \leq k$, can be decreased by only firing transitions in $F$ (since $F$ is a $T$-subnet, and it is structurally deadlockable) until $F$ deadlocks. Moreover, no deleted input transition of any $N_{i}$ is a transition of $F$; thus, by homogeneity, one can fire in $F$ while never firing such inputs of $N_{i}$, and new tokens will not be produced in any $N_{i}$ in the process. We deduce that a firing sequence always exists that deadlocks all $N_{i}$ 's first, then deadlocks $F$, reaching a global deadlock.

Figure 6 depicts three quasi strongly connected HJF nets ${ }^{5}$. The first one is structurally live and not sub-consistent; the second and the third ones are sub-consistent, thus structurally deadlockable.

We are now able to deduce the next corollary for structural liveness.

Corollary 1 (Structural liveness). Consider a quasi strongly connected HJF net. It is structurally live if and only if it is not sub-consistent.

Proof. If such a net is sub-consistent, then it is structurally deadlockable by Theorem 1, thus not structurally live. For the converse, if it is not structurally live, it is structurally deadlockable (Lemmas 3 and 4) and sub-consistent by Lemma 2.

\footnotetext{
${ }^{5}$ Each of them has traps and is weakly sur-consistent (i.e. structurally repetitive).
} 

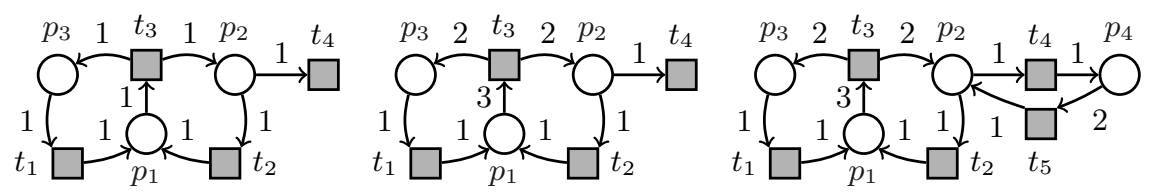

Fig. 6. Three quasi strongly connected HJF nets are pictured. On the left, it is not sub-consistent, hence it is structurally live (a token in $p_{1}$ yields liveness). The nets in the middle and on the right are sub-consistent. In both of them, there is a structurally deadlockable FA T subnet: the T-subnet $F$ induced by $t_{4}$ in the middle, the T-subnet $F^{\prime}$ induced by $\left\{t_{4}, t_{5}\right\}$ on the right. In the proof, after the deletion of $F$ and $F^{\prime}$ with their outgoing transitions, the cycle $t_{1} p_{1} t_{3} p_{3}$ remains in both nets. This cycle is strongly connected and sub-consistent, thus it can deadlock; when firings in this cycle occur in the initial nets (before deletion), tokens are produced only in $p_{2}$, and $t_{2}$ shall never be fired. After the cycle deadlocks, $F$ and $F^{\prime}$ deadlock, inducing a global deadlock.

Polynomial-time complexity. Checking the existence of a rational solution to $\mathcal{I} \cdot Y \lesseqgtr \mathbb{O}, Y \geq \mathbb{1}$, and computing one when it exists, can be done in weakly polynomial time with linear programming. Multiplying this solution by an adequate rational number, we get an integer solution. Thus, Theorem 1 and Corollary 1 can be checked in polynomial time.

\subsection{Generalization of the Conditions to all HJF Nets}

A simple necessary condition for structural deadlockability is stated next.

Lemma 14. If a net $(P, T, W)$ is structurally deadlockable, then $P$ is a siphon.

Proof. If $P$ is not a siphon, then ${ }^{\bullet} P \nsubseteq P^{\bullet}$, meaning that some input transition of some place in $P$ has no input place: such a transition is always fireable, contradicting deadlockability. Thus, $P$ is a siphon.

In Theorem 1 and Corollary 1, the nets considered are quasi strongly connected. Their set of places is their unique siphon by Lemma 1 . We generalize Theorem 1 and Corollary 1 through the two following corollaries.

Corollary 2 (Structural deadlockability). Consider a connected HJF net $N=(P, T, W)$. Denote by $C$ the set of all the maximal quasi strongly connected subnets of $N$ that contain at least one place and one transition. $N$ is structurally deadlockable if and only if $P$ is a siphon and each element of $C$ is sub-consistent.

Proof. Assume that $N$ is structurally deadlockable. $P$ is a siphon by Lemma 14 . Consider a non-sub-consistent element $c$ of $C$. By Corollary 1, $c$ is structurally live. In $N$, the outputs of nodes of $c$ that do not belong to $c$ may only be places while the inputs of nodes of $c$ that do not belong to $c$ may only be transitions: in the first case, each output transition belongs to $c$ since $c$ is a maximal quasi strongly connected subnet; in the second case, an input place would be the unique input of a transition $t$, such that $t$ has no input in $c$, contradicting the definition of $c$. Thus, $c$ remains structurally live in $N$, contradicting deadlockability. 
Conversely, each element of $c$ is sub-consistent, hence structurally deadlockable (Theorem 1 ). For each place $p$ of $N$, either $p$ has no output, or the maximal quasi strongly connected subnet containing $p$ has a transition, belongs to $C$ and is structurally deadlockable. Since $P$ is a siphon, each transition of $N$ has an input. Hence, for every marking $M_{0},\left(N, M_{0}\right)$ can be deadlocked by following the paths of the reduced graph $R$ of $N$, since each node of $R$ denotes either a place with no output or a subnet of an element of $C$.

Corollary 3 (Structural liveness). Let $N=(P, T, W)$ be a connected HJF net. Let $C$ be the set of maximal quasi strongly connected subnets of $N$ with no input node in $N$ and containing a place and a transition. Then, $N$ is structurally live if and only if no element of $C$ is sub-consistent.

Proof. If an element of $C$ is sub-consistent, it is structurally deadlockable (Theorem 1) with no input, hence $N$ cannot be structurally live. Conversely, if no element of $C$ is sub-consistent, each is structurally live (Corollary 1). Thus, there exists a marking for which all elements of $C$ are live. Such live subsystems can generate an arbitrarily large number of tokens in their output places in $N$. There may also exist nodes without input, which are necessarily single transitions, hence also live subsystems. By join-freeness and homogeneity, an arbitrarily large number of tokens can reach each place of the system by following the directed paths in the reduced graph of $N$. We deduce liveness.

Polynomial-time complexity. The set of the maximal quasi strongly connected subnets of a join-free net forms a partition (or disjoint covering) of its nodes. Thus, the number of these subnets is linear in the size of the system. Determining these subnets and checking their sub-consistency are polynomial-time problems, and the conditions of Theorem 1 and Corollary 1 can be checked in polynomial-time.

\section{Non-monotonic Liveness of Inhomogeneous JF Nets}

Liveness is not always monotonic, as shown for a bounded HAC system in [8]. In HFC (hence also OFC and HJF) nets and in some other classes, liveness is known to be monotonic $[5,22,16]$. For the inhomogeneous join-free class, we present on the left of Figure 7 a simple example of non-monotonic liveness under the strong connectedness assumption. On the right of the same figure, we provide, as far as we know, the first structurally bounded, live, non m-live join-free system.

\section{Improvements on a Previous Work on HAC Nets}

We focus here on results of [11]. First, we observe that the pureness assumption used in [11], which forbids nodes $x$ such that ${ }^{\bullet} x \cap x^{\bullet} \neq \emptyset$, is not necessary. Then, we exhibit an incorrect argument of a proof in one of the central theorems of the same paper, which is based on quasi strongly connected HJF subnets. Finally, we correct this proof using Theorem 1. 

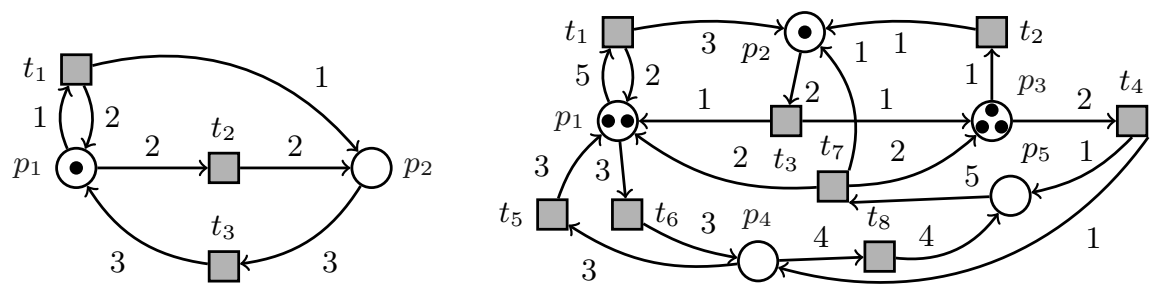

Fig. 7. The live and unbounded JF system on the left is not $\mathrm{m}$-live, since adding a token to $p_{1}$ allows to fire $t_{2}$, leading to a deadlock. On the right, the JF system is strongly connected and live; however, it is not m-live, since adding a token to $p_{3}$ and firing two times $t_{4}$ leads to a deadlock.

Unnecessary pureness. The assumption of pureness, which forbids self-loops, is only exploited in the proof of the very first lemma of [11] (Lemma 3.1), which is used in various later occasions. By refining a bit the proof, one can get rid of this precondition.

An incorrect argument in a previous proof for HAC nets. Consider the next characterization of [11] for the m-live and bounded markings of HAC nets, where $N_{D}$ denotes the P-subnet of $N$ induced by the set $D$ of places and $M_{0}^{D}$ is the restriction $\left.M_{0}\right|_{D}$.

Theorem 2 (Theorem 5.2 in [11]). An HAC system $\left(N, M_{0}\right)$ is monotonically live and bounded if and only if every place $p$ is covered by a minimal siphon, and for every minimal siphon $D,\left(N_{D}, M_{0}^{D}\right)$ is live and bounded.

Let us exhibit the problem that appears in the proof of this theorem. The paper correctly shows the "if" part, i.e. that the liveness and boundedness of all the P-subsystems induced by all the minimal siphons, which cover all places, implies the monotonic liveness and boundedness of the entire HAC system. It also proves that if $\left(N, M_{0}\right)$ is live and bounded, then every place is covered by a minimal siphon, and that if $N$ is live from each marking $M \geq M_{0}$ then $\left(N_{D}, M_{0}^{D}\right)$ is live for every minimal siphon $D$. However, to show that the existence of a minimal siphon $D$ such that $\left(N_{D}, M_{0}^{D}\right)$ is live and unbounded implies that $\left(N, M_{0}\right)$ is not monotonically bounded, the authors use the next argument:

If $p$ is unbounded in $\left(N_{D}, M_{0}^{D}\right)$, for each integer $k$ there is a firing sequence $\sigma_{k}$ such that $M_{0}^{D}\left[\sigma_{k}\right\rangle M_{k}$ in $N_{D}$ with $M_{k}(p)>k$. Then there exist $M \geq M_{0}$ and $M^{\prime}$ such that, in $N, M\left[\sigma_{k}\right\rangle M^{\prime}$ and $M^{\prime}(p)=M_{k}(p)>k$, contradicting the boundedness of $(N, M)$.

This argument is incorrect, since it considers that $\exists p \in S, \forall k \in \mathbb{N}, \exists M \geq M_{0}$, $\exists M^{\prime} \in[M\rangle: M^{\prime}(p)>k$ instead of the desired goal, which is: $\exists p \in S, \exists M \geq M_{0}$, $\forall k \in \mathbb{N}, \exists M^{\prime} \in[M\rangle: M^{\prime}(p)>k$. Actually, the authors do not use there the fact that $D$ is a minimal siphon. However, we are going to show that the authors had 
a correct intuition and the property they claim is valid. Other properties of the literature for HAC nets, like [3,1], do not help.

From Theorem 3.2 in the same paper [11], we know that $\left(N_{D}, M_{0}^{D}\right)$ is live. Let us now proceed by contradiction and assume that $\left(N_{D}, M_{0}^{D}\right)$ is unbounded. Since it is known that live and bounded Petri nets are consistent [18], the net $N$ is consistent. Hence, every P-subnet induced by any subset of places is also consistent. In particular, this is also the case for $N_{D}$. If a system is unbounded, the underlying net is not structurally bounded. We recall the next characterization for this property, which can be found in various studies, e.g. in [18].

Lemma 15 (Corollary 16 in [18]). A net with incidence matrix $\mathcal{I}$ is not structurally bounded if and only if there exists a vector $X \geqslant \mathbb{O}$ such that $\mathcal{I} \cdot X \geqslant \mathbb{0}$.

Applying Lemma 11, we get the sub-consistency and sur-consistency of $N_{D}$.

Each minimal siphon of an HAC net induces a quasi strongly connected HJF P-subnet (by [11] and Lemma 1). Applying Theorem $1, N_{D}$ is structurally deadlockable, contradicting the liveness of $\left(N_{D}, M_{0}^{D}\right)$, which must thus be bounded. We deduce the validity of Theorem 5.2 in [11].

\section{Conclusion and Perspectives}

We examined a crucial substructure of several complex subclasses of Petri nets, the weighted join-free class. In the homogeneous case, we obtained polynomialtime characterizations of structural deadlockability and structural liveness that are not subsumed by previous known methods. They enrich the set of efficient structural analysis techniques for weighted Petri nets. In the inhomogeneous, structurally bounded join-free case, we showed the non-monotonicity of liveness. Finally, we used our new structural conditions on the homogeneous join-free class to correct a previous erroneous proof of a characterization of monotonic liveness and boundedness in the homogeneous asymmetric-choice class.

An important perspective is to find out, for the class of Petri net systems that are decomposable into monotonically live siphon-induced join-free P-subsystems, under which conditions such local behaviors propagate to the entire system. A complementary objective is to broaden the methods that check decomposability in polynomial-time and to deduce efficient sufficient conditions of monotonic liveness and boundedness for larger classes. Future applications of such methods encompass notably the design of embedded systems.

\section{References}

1. Barkaoui, K., Couvreur, J.M., Klai, K.: On the Equivalence between Liveness and Deadlock-freeness in Petri nets. In: Ciardo, G., Darondeau, P. (eds.) Applications and Theory of Petri Nets, LNCS, vol. 3536, pp. 90-107 (2005)

2. Barkaoui, K., Minoux, M.: A polynomial-time graph algorithm to decide liveness of some basic classes of bounded Petri nets. In: Jensen, K. (ed.) Application and Theory of Petri Nets, LNCS, vol. 616, pp. 62-75 (1992) 
3. Barkaoui, K., Pradat-Peyre, J.F.: On liveness and controlled siphons in Petri nets, LNCS, vol. 1091, pp. 57-72 (1996)

4. Cheng, A., Esparza, J., Palsberg, J.: Complexity results for 1-safe nets. In: Shyamasundar, R. (ed.) Foundations of Software Technology and Theoretical Computer Science, LNCS, vol. 761, pp. 326-337 (1993)

5. Desel, J., Esparza, J.: Free Choice Petri Nets, Cambridge Tracts in Theoretical Computer Science, vol. 40. Cambridge University Press, New York, USA (1995)

6. Esparza, J.: Decidability and complexity of Petri net problems-an introduction. In: Reisig, W., Rozenberg, G. (eds.) Lectures on Petri Nets I: Basic Models, LNCS, vol. 1491, pp. 374-428 (1998)

7. Esparza, J., Nielsen, M.: Decidability issues for Petri nets-a survey. BRICS Report Series 1(8) (1994)

8. Heiner, M., Mahulea, C., Silva, M.: On the Importance of the Deadlock Trap Property for Monotonic Liveness. In: International Workshop on Biological Processes and Petri nets (BioPPN), A satellite event of Petri Nets 2010 (2010)

9. Hujsa, T., Delosme, J.M., Munier-Kordon, A.: Polynomial sufficient conditions of well-behavedness and home markings in subclasses of weighted Petri nets. Transactions on Embedded Computing Systems (2014)

10. Hujsa, T., Delosme, J.M., Munier-Kordon, A.: On liveness and reversibility of equal-conflict Petri nets. Fundamenta Informaticae 146(1), 83-119 (2016)

11. Jiao, L., Cheung, T.Y., Lu, W.: On liveness and boundedness of Asymmetric Choice nets. Theoretical Computer Science 311(1-3), 165-197 (2004)

12. Lee, E.A., Messerschmitt, D.G.: Synchronous Data Flow. Proceedings of the IEEE 75(9), 1235-1245 (1987)

13. Lipton, R.: The reachability problem requires exponential space. Tech. Rep. 62, Department of Computer Science, Yale University (1976)

14. Mayr, E.W., Weihmann, J.: Results on equivalence, boundedness, liveness, and covering problems of BPP-Petri nets. In: Application and Theory of Petri Nets and Concurrency. pp. 70-89 (2013)

15. Mayr, E.W., Weihmann, J.: Complexity results for problems of communication-free Petri nets and related formalisms. Fundamenta Informaticae 137(1), 61-86 (2015)

16. Recalde, L., Teruel, E., Silva, M.: Modeling and analysis of sequential processes that cooperate through buffers. IEEE Transactions on Robotics and Automation 14(2), 267-277 (1998)

17. Sifakis, J.: Structural properties of Petri nets. In: Winkowski, J. (ed.) Mathematical Foundations of Computer Science, LNCS, vol. 64, pp. 474-483 (1978)

18. Silva, M., Teruel, E., Colom, J.M.: Linear algebraic and linear programming techniques for the analysis of place/transition net systems. In: Reisig, W., Rozenberg, G. (eds.) Lectures on Petri Nets I: Basic Models, LNCS, vol. 1491, pp. 309-373 (1998)

19. Teruel, E.: Structure Theory of Weighted Place/Transition Net Systems: The Equal Conflict Hiatus. Ph.D. thesis, DIEI. University of Zaragoza, Spain (1994)

20. Teruel, E., Colom, J.M., Silva, M.: Choice-Free Petri nets: a model for deterministic concurrent systems with bulk services and arrivals. IEEE Transactions on Systems, Man and Cybernetics, Part A 27(1), 73-83 (1997)

21. Teruel, E., Silva, M.: Liveness and home states in Equal Conflict systems. In: Marsan, M.A. (ed.) Application and Theory of Petri Nets 1993, LNCS, vol. 691, pp. 415-432 (1993)

22. Teruel, E., Silva, M.: Structure theory of Equal Conflict systems. Theoretical Computer Science 153(1\&2), 271-300 (1996) 